\title{
A NOTE ON THE INTERFACE DYNAMICS FOR CONVECTION IN POROUS MEDIA
}

\author{
DIEGO CÓRDOBA, FRANCISCO GANCEDO AND RAFAEL ORIVE
}

\begin{abstract}
We study the fluid interface problem through porous media given by two incompressible 2-D fluids of different densities. This problem is mathematically analogous to the dynamics interface for convection in porous media, where the free boundary evolves between fluids with different temperature. We find a new formula for the evolution equation of the free boundary parameterized as a function in the periodic case. In this formula there is no a principal value in the non-local integral operator involved in the equation, giving a simpler system. Using this formulation, we perform numerical simulations in the stable case (denser fluid below) which show a strong regularity effect in the periodic interface.
\end{abstract}

\section{INTRODUCTION}

In this paper we study the evolution of a sharp interface between fluids of different densities in a two dimensional incompressible flow through porous media. Fluid interface problems in porous media have been widely considered (see [10, 16]), together with the two-phase Hele-Shaw flow (see [11, 3]).

The motion of a 2-D flow in a Hele-Shaw cell [12 consists of the dynamics of a fluid trapped between two fixed parallel plates close enough together, so that the fluid essentially only moves in two directions. The evolution of 2-D incompressible fluids in a porous medium becomes mathematically analogous to that of Hele-Shaw cell, since the dynamics are modeled by the following momentum equation:

$$
\frac{\mu}{a} v(x, y, t)=-\nabla p(x, y, t)-(0, g \rho(x, y, t))
$$

where $v$ is the velocity, $p$ is the pressure, $\mu$ is the dynamic viscosity, $\rho$ is the liquid density, and $g$ is the acceleration due to gravity. We take $x, y \in \mathbb{R}$ the spatial variables and $t \geq 0$ the time. The constant $a$ corresponds to the permeability of the isotropic medium in dynamics in porous media, being the equation (1) the well-known Darcy's law [2]. In a Hele-Shaw cell, the constant $a$ is equal to $b^{2} / 12$, with $b$ the distance between the plates.

The evolution of an interface between two fluids in a porous medium with different viscosities, $\mu_{1}, \mu_{2}$, and densities, $\rho_{1}, \rho_{2}$, was modeled using Darcy's law by Muskat in [14]. Saffman and Taylor in [15] studied the problem proposed by Muskat noting the analogies with the two-phase Hele-Shaw flow.

Date:

Key words and phrases. Porous media, fluid interface, incompressible flow.

2000 Mathematics Subject Classification. 76S05, 76B03, 65N06.

The authors were partially supported by the grant MTM2005-05980 of the MEC (Spain). The two first authors were partially supported by the grant PAC-05-005-2 of the JCLM (Spain). The third author was partially supported by the grant MTM2005-00714 of the MEC (Spain). 
Both free boundary problems can be modeled using the Laplace-Young condition, so that the pressure of the fluid across the interface is given by a jump discontinuity that is equal to the local curvature times the surface tension. With surface tension, the problems have classical solutions (see [11]).

The case without surface tension was studied by Siegel, Caflisch and Howison in [16]. They proved global-in-time existence for near planar initial data in a stable case when the Atwood number

$$
A_{\mu}=\frac{\mu_{1}-\mu_{2}}{\mu_{1}+\mu_{2}},
$$

is positive. They showed ill-posedness in a unstable case when $A_{\mu}$ is negative.

In the same year, Ambrose in [1] treated the problem with initial data fulfilling

$$
\left(\rho_{2}-\rho_{1}\right) \mathrm{g} \cos (\theta(\alpha, 0))+2 A_{\mu} U(\alpha, 0)>0,
$$

where the curve $(x(\alpha, t), y(\alpha, t))$ is the interface, $\theta$ is the angle that the tangent to the curve forms with the horizontal, and $U$ is the normal velocity (given by the Birkhoff-Rott integral).

We consider the case of two fluids that have equal viscosity, $A_{\mu}=0$, so that the interface is between fluids of different densities. This equation has been considered in a different frame in convection in porous media, where the scalar $\rho$ plays the role of the temperature. In [10, Dombre, Pumir and Siggia consider the following transport equation for the temperature $T$ :

$$
T_{t}+v \cdot \nabla T=0,
$$

with an incompressible velocity given by

$$
v(x, y, t)=(0, T(x, y, t))-\nabla p(x, y, t),
$$

where the active scalar $T$ has a jump across an interface. They study the case of analytic initial interface, performing analytic continuation of the contour equation. They consider meromorphic initial conditions with complex poles, and treat the dynamics of these critical points. If a pole reaches the real axis, then it becomes a physical singularity. The case studied in that paper corresponds to the unstable case which we discuss below in section 3 .

In this work, we consider the following evolution problem for the active scalar $\rho$

$$
\rho_{t}+v \cdot \nabla \rho=0,
$$

with an incompressible velocity given by

$$
v(x, y, t)=-\nabla p(x, y, t)-(0, \rho(x, y, t)),
$$

where we take in this case $\mu / a=g=1$ in Darcy's law without loss of generality.

In a recent work (see [8]) we have considered the case of smooth initial density for the equations (2) and (3), obtaining well-posedness, global existence criteria, blow-up for solutions of infinite energy, and performing numerical simulations. These numerical simulations together with the analytical blow-up criteria show no evidence of singularities.

Here $\rho$ satisfies (2) in a weak sense and is defined by

$$
\rho(x, y, t)=\left\{\begin{array}{lll}
\rho_{1} & \text { in } \quad \Omega_{1}(t)=\{y>f(x, t)\}, \\
\rho_{2} & \text { in } \quad \Omega_{2}(t)=\mathbb{R}^{2}-\Omega_{1}(t),
\end{array}\right.
$$

where $\rho_{1}, \rho_{2} \geq 0$ are different constants and $(\alpha, f(\alpha, t))$ is the interface. We study the periodic case, that is $f(\alpha+2 \pi, t)=f(\alpha, t)$.

It is well-known (see [13]) that for these contour dynamics systems in incompressible fluid, the velocity in the tangential direction does not modify the shape of the interface. Changes 
in the tangential component of the velocity only introduce changes in the parametrization of the curve. In [6] two of the authors use this property to parameterize the interface as a function $(\alpha, f(\alpha, t))$, getting the following evolution equation

$$
\begin{aligned}
f_{t}(\alpha, t) & =\frac{\rho_{2}-\rho_{1}}{2 \pi} P V \int_{\mathbb{R}} \frac{\left(\partial_{\alpha} f(\alpha, t)-\partial_{\alpha} f(\alpha-\beta, t)\right) \beta}{\beta^{2}+(f(\alpha, t)-f(\alpha-\beta, t))^{2}} d \beta, \\
f(\alpha, 0) & =f_{0}(\alpha), \quad \alpha \in \mathbb{R} .
\end{aligned}
$$

The periodicity of $f(\alpha, t)$ makes the principal value (see [17]) crucial in order to understand the above formulation. We also get an equivalent equation given by

$$
\begin{aligned}
f_{t}(\alpha, t)= & \frac{\rho_{2}-\rho_{1}}{2 \pi} \int_{\mathbb{T}} \frac{\left(\partial_{\alpha} f(\alpha, t)-\partial_{\alpha} f(\alpha-\beta, t)\right) \beta}{\beta^{2}+(f(\alpha, t)-f(\alpha-\beta, t))^{2}} P(\beta, f(\alpha, t)-f(\alpha-\beta, t)) d \beta \\
& +\frac{\rho_{2}-\rho_{1}}{2 \pi} \int_{\mathbb{T}}\left(\partial_{\alpha} f(\alpha, t)-\partial_{\alpha} f(\alpha-\beta, t)\right) Q(\beta, f(\alpha, t)-f(\alpha-\beta, t)) d \beta,
\end{aligned}
$$

with

$$
\begin{aligned}
& P(x, y) \in C_{c}^{\infty}(\mathbb{T} \times \mathbb{R}), \quad P \geq 0, \quad \text { supp } P \subset\left\{x^{2}+y^{2} \leq 4\right\}, \\
& P=1 \text { in }\left\{x^{2}+y^{2} \leq 1\right\} \quad P(-x,-y)=P(x, y),
\end{aligned}
$$

and

$$
Q(x, y) \in C_{b}^{\infty}(\mathbb{T} \times \mathbb{R}), \quad Q(0,0)=0 .
$$

In this system a principal value is not necessary in order to understand the non-local operator involved in the formulation, but the functions $P$ and $Q$ are unknown. When the denser fluid is below, $\rho_{2}>\rho_{1}$, we prove local existence and uniqueness for the system (5), so that the problem is well-posed. When the denser fluid is above, $\rho_{2}<\rho_{1}$, we prove that (4) is ill-posed. We obtain this result in a similar way as in [16], using global solutions of (4) in the stable case, $\rho_{2}>\rho_{1}$, for small initial data.

In this work we find the explicit formula for the evolution equation (5) given by

$$
\begin{aligned}
& f_{t}(\alpha, t)=\frac{\rho_{2}-\rho_{1}}{4 \pi} \int_{\mathbb{T}} \frac{\left(\partial_{\alpha} f(\alpha, t)-\partial_{\alpha} f(\alpha-\beta, t)\right) \tan (\beta / 2)}{\tan ^{2}(\beta / 2)+\tanh ^{2}((f(\alpha, t)-f(\alpha-\beta, t)) / 2)} d \beta \\
& \quad-\frac{\rho_{2}-\rho_{1}}{4 \pi} \int_{\mathbb{T}}\left(\partial_{\alpha} f(\alpha, t)-\partial_{\alpha} f(\alpha-\beta, t)\right) \frac{\tan (\beta / 2) \tanh ^{2}((f(\alpha, t)-f(\alpha-\beta, t)) / 2)}{\tan ^{2}(\beta / 2)+\tanh ^{2}((f(\alpha, t)-f(\alpha-\beta, t)) / 2)} d \beta .
\end{aligned}
$$

We use this equation to get decay estimates of the $L^{\infty}$ norm of the interface for the stable case for a more general class of initial data than in [7]. This new system lets us perform numerical simulations with the exact equation in a simpler formulation, without a principal value at infinity.

The paper is organized as follows. In section 2 we derive the new formula for the contour equation. In section 3 we show the main ideas of why in the unstable case (the denser fluid is above) the problem is ill-posed. In section 4 we study the stable case. We prove local existence and uniqueness of a simpler model of the contour equation. We give the maximum principle for the interface, and we present decay estimates of the $L^{\infty}$ norm. We perform numerical simulations in which the analytical properties are satisfied and a smoothing effect appears in the equation. Finally, we present the conclusions in section 5. 


\section{The Contour Equation}

The goal of this section is to find the following formulation for the free boundary problem

(6)

$$
\begin{aligned}
& f_{t}(\alpha, t)=\bar{\rho} \int_{\mathbb{T}} \frac{\left(\partial_{\alpha} f(\alpha, t)-\partial_{\alpha} f(\alpha-\beta, t)\right) \tan (\beta / 2)\left(1-\tanh ^{2}((f(\alpha, t)-f(\alpha-\beta, t)) / 2)\right)}{2 \pi\left(\tan ^{2}(\beta / 2)+\tanh ^{2}((f(\alpha, t)-f(\alpha-\beta, t)) / 2)\right)} d \beta, \\
& f(\alpha, 0)=f_{0}(\alpha),
\end{aligned}
$$

with $\bar{\rho}=\left(\rho_{2}-\rho_{1}\right) / 2$. We consider the equation for two fluids with different densities, so $\rho$ is given by

$$
\rho(x, y, t)= \begin{cases}\rho_{1}, & \Omega_{1}(t), \\ \rho_{2}, & \Omega_{2}(t)=\mathbb{R}^{2}-\Omega_{1}(t),\end{cases}
$$

where the interface $\partial \Omega_{1}(t)$ is parameterized by $z(\alpha, t)=\left(z_{1}(\alpha, t), z_{2}(\alpha, t)\right)$. Using Darcy's law (3), we find the vorticity given by

$$
w(x, y, t)=-\partial_{x} \rho(x, y, t) .
$$

There is a jump of densities, so $\partial_{x} \rho=2 \bar{\rho} \partial_{\alpha} z_{2}(\alpha, t) \delta((x, y)-z(\alpha, t))$. Biot-Savart law yields

$$
v(x, y, t)=-\frac{\bar{\rho}}{\pi} P V \int_{\mathbb{R}} \frac{\left(z_{2}(\beta, t)-y, x-z_{1}(\beta, t)\right)}{|(x, y)-z(\beta, t)|^{2}} \partial_{\alpha} z_{2}(\beta, t) d \beta,
$$

for $(x, y) \neq z(\alpha, t)$. Taking $(x, y) \rightarrow z(\alpha, t)$, we find that the velocity is discontinuous in the tangential direction, but we can write

$$
z_{t}(\alpha, t)=-\frac{\bar{\rho}}{\pi} P V \int_{\mathbb{R}} \frac{(z(\alpha, t)-z(\beta, t))^{\perp}}{|z(\alpha, t)-z(\beta, t)|^{2}} \partial_{\alpha} z_{2}(\beta, t) d \beta,
$$

if the tangential terms are ignored. Writing $z(\alpha, t)=z_{1}(\alpha, t)+i z_{2}(\alpha, t)$, we find

$$
z_{t}^{*}(\alpha, t)=-\frac{\bar{\rho}}{\pi i} P V \int_{\mathbb{R}} \frac{1}{z(\alpha, t)-z(\beta, t)} \partial_{\alpha} z_{2}(\beta, t) d \beta .
$$

We consider the periodic case, then $z(\alpha+2 \pi k, t)=z(\alpha, t)+2 \pi k$. Using the following identity for complex numbers

$$
\frac{1}{\pi}\left(\frac{1}{z}+\sum_{k \geq 1} \frac{2 z}{z^{2}-(2 \pi k)^{2}}\right)=\frac{1}{2 \pi \tan (z / 2)},
$$

and splitting the integral in (7), we obtain

$$
z_{t}^{*}(\alpha, t)=-\frac{\bar{\rho}}{2 \pi i} P V \int_{\mathbb{T}} \frac{1}{\tan ((z(\alpha, t)-z(\beta, t)) / 2)} \partial_{\alpha} z_{2}(\beta, t) d \beta .
$$

It yields (ignoring the time dependence)

$$
\partial_{t} z_{1}(\alpha)=\bar{\rho} P V \int_{\mathbb{T}} \partial_{\alpha} z_{2}(\beta) \frac{\tanh \left(\left(z_{2}(\alpha)-z_{2}(\beta)\right) / 2\right)\left(1+\tan ^{2}\left(\left(z_{1}(\alpha)-z_{1}(\beta)\right) / 2\right)\right)}{2 \pi\left(\tan ^{2}\left(\left(z_{1}(\alpha)-z_{1}(\beta)\right) / 2\right)+\tanh ^{2}\left(\left(z_{2}(\alpha)-z_{2}(\beta)\right) / 2\right)\right)} d \beta
$$

and

$$
\left.\partial_{t} z_{2}(\alpha)=\bar{\rho} P V \int_{\mathbb{T}} \partial_{\alpha} z_{2}(\beta) \frac{\tan \left(\left(z_{1}(\alpha)-z_{1}(\beta)\right) / 2\right)\left(\tanh ^{2}\left(\left(z_{2}(\alpha)-z_{2}(\beta)\right) / 2\right)-1\right)}{2 \pi\left(\tan ^{2}\left(\left(z_{1}(\alpha)-z_{1}(\beta)\right) / 2\right)+\tanh ^{2}\left(\left(z_{2}(\alpha)-z_{2}(\beta)\right) / 2\right)\right)}\right) d \beta .
$$


We can change the tangential velocity without altering the shape of the interface, so

$$
z_{t}(\alpha, t)=\bar{\rho} P V \int_{\mathbb{T}} \partial_{\alpha} z_{2}(\beta, t)(\quad, \quad) d \beta+c(\alpha, t) \partial_{\alpha} z(\alpha, t) .
$$

We know that the interface can be parameterized as a function (see [6]). In order to get this, we need to show that $\partial_{t} z_{1}(\alpha, t)=0$ and that if the initial interface is given by $z(\alpha, 0)=$ $\left(\alpha, f_{0}(\alpha)\right)$ then we get $z(\alpha, t)=(\alpha, f(\alpha, t))$. We choose

$$
c(\alpha, t)=-\bar{\rho} P V \int_{\mathbb{T}} \partial_{\alpha} f(\beta, t) \frac{\tanh ((f(\alpha, t)-f(\beta, t)) / 2)\left(1+\tan ^{2}((\alpha-\beta) / 2)\right)}{2 \pi\left(\tan ^{2}((\alpha-\beta) / 2)+\tanh ^{2}((f(\alpha, t)-f(\beta, t)) / 2)\right)} d \beta,
$$

and the evolution equation follows

$$
\begin{aligned}
& f_{t}(\alpha, t)=\bar{\rho} P V \int_{\mathbb{T}} \partial_{\alpha} f(\beta, t) \frac{\tan ((\alpha-\beta) / 2)\left(\tanh ^{2}((f(\alpha, t)-f(\beta, t)) / 2)-1\right)}{2 \pi\left(\tan ^{2}((\alpha-\beta) / 2)+\tanh ^{2}((f(\alpha, t)-f(\beta, t)) / 2)\right)} d \beta \\
& -\bar{\rho} P V \int_{\mathbb{T}} \partial_{\alpha} f(\beta, t) \frac{\tanh ((f(\alpha, t)-f(\beta, t)) / 2)\left(1+\tan ^{2}((\alpha-\beta) / 2)\right)}{2 \pi\left(\tan ^{2}((\alpha-\beta) / 2)+\tanh ^{2}((f(\alpha, t)-f(\beta, t)) / 2)\right)} d \beta \partial_{\alpha} f(\alpha, t) .
\end{aligned}
$$

If we defined the functions $G$ and $H$ by

$$
\begin{aligned}
& G(\alpha, \beta)=\sin ^{2}((\alpha-\beta) / 2) \cosh ^{2}((f(\alpha, t)-f(\beta, t)) / 2) \\
& H(\alpha, \beta)=\cos ^{2}((\alpha-\beta) / 2) \sinh ^{2}((f(\alpha, t)-f(\beta, t)) / 2) .
\end{aligned}
$$

Then the following identity

$$
\int_{\mathbb{T}} \partial_{\beta} \ln (G(\alpha, \beta)+H(\alpha, \beta)) d \beta=0,
$$

shows that

$$
\begin{gathered}
-P V \int_{\mathbb{T}} \partial_{\alpha} f(\beta, t) \frac{\tanh ((f(\alpha, t)-f(\beta, t)) / 2)\left(1+\tan ^{2}((\alpha-\beta) / 2)\right)}{\tan ^{2}((\alpha-\beta) / 2)+\tanh ^{2}((f(\alpha, t)-f(\beta, t)) / 2)} d \beta= \\
P V \int_{\mathbb{T}} \frac{\tan ((\alpha-\beta) / 2)\left(1-\tanh ^{2}((f(\alpha, t)-f(\beta, t)) / 2)\right)}{\tan ^{2}((\alpha-\beta) / 2)+\tanh ^{2}((f(\alpha, t)-f(\beta, t)) / 2)} d \beta .
\end{gathered}
$$

Using the above equality in (8) we obtain

$$
f_{t}(\alpha, t)=\bar{\rho} \int_{\mathbb{T}} \frac{\left(\partial_{\alpha} f(\alpha, t)-\partial_{\alpha} f(\beta, t)\right) \tan ((\alpha-\beta) / 2)\left(1-\tanh ^{2}((f(\alpha, t)-f(\beta, t)) / 2)\right)}{2 \pi\left(\tan ^{2}((\alpha-\beta) / 2)+\tanh ^{2}((f(\alpha, t)-f(\beta, t)) / 2)\right)} d \beta
$$

and a change of variables gives (6). We would like to point out that the above formula does not have a principal value for $\beta=\alpha$ and a priori it is more regular than (7).

\section{Unstable CASE}

In this section we present some ideas of the reasons why for $\bar{\rho}<0$ the system (6) is ill-posed. Using the Fourier transform, we define the Sobolev space $H^{s}$ with norm

$$
\|f\|_{H^{s}}^{2}=\sum_{\xi \in \mathbb{Z}}\left(1+|\xi|^{2 s}\right)|\hat{f}(\xi)|^{2} .
$$

In [6] can be found the following result: 
Theorem 3.1. If $s>3 / 2$, then for any $f_{0} \in H^{s}$, the system (4) is ill-posed for $\bar{\rho}<0$; i.e. for any $\varepsilon>0$ there exists a solution $f$ of (4) and $0<\delta<\varepsilon$ such that $\left\|f_{0}\right\|_{H^{s}} \leq \varepsilon$ and $\|f\|_{H^{s}}(\delta)=\infty$.

Remark 3.2. Ignoring the terms of order two in (4) or (6), we conclude that the small solution $f(\alpha, t)$ satisfies

$$
\begin{aligned}
f_{t}(\alpha, t) & =-\bar{\rho} \Lambda f(\alpha, t), \\
f(\alpha, 0) & =f_{0}(\alpha),
\end{aligned}
$$

where the operator $\Lambda$ is defined by $\Lambda=(-\Delta)^{1 / 2}$. By taking the Fourier transform, we get

$$
\hat{f}(\xi)=\hat{f}_{0}(\xi) e^{-\bar{\rho}|\xi| t}
$$

and we obtain an ill-posed problem for $\bar{\rho}<0$ with general initial data in Sobolev spaces.

The system (6) can be written as

$$
f_{t}(\alpha, t)=-\bar{\rho} \Lambda f(\alpha, t)+T(f)(\alpha, t) .
$$

Considering the stable case $\bar{\rho}>0$, if we take near planar initial data, i.e.

$$
\sum|\xi|\left|\hat{f}_{0}(\xi)\right|<<1
$$

applying Fourier techniques we get that the dissipative term $\Lambda$ controls the nonlinear operator $T$ and we can obtain global-in-time solutions. In fact, we can extend these solution analytically on the strip $|\Im z|<c t$ with the constant $c$ fixed.

Making a change of variables, we define

$$
f_{\lambda}(\alpha, t)=\lambda^{-1} f\left(\lambda \alpha,-\lambda t+\lambda^{1 / 2}\right)
$$

obtaining $\left\{f_{\lambda}\right\}_{\lambda>0}$ a family of solutions to the unstable case. If we take initial data satisfying

$$
\sum|\xi|^{1+\gamma}\left|\hat{f}_{0}(\xi)\right|<C
$$

and

$$
\sum|\xi|^{1+\gamma+\zeta}\left|\hat{f}_{0}(\xi)\right|=\infty
$$

for $\gamma, \zeta>0$, using the dissipation and the analyticity of the solutions we obtain

$$
\begin{aligned}
\left\|f_{\lambda}\right\|_{H^{s}}(0) & =|\lambda|^{s-\frac{3}{2}}\|f\|_{H^{s}}\left(\lambda^{1 / 2}\right) \\
& \leq C|\lambda|^{s-\frac{3}{2}} \sum e^{|\xi|}\left|\hat{f}\left(\xi, \lambda^{1 / 2}\right)\right| \\
& \leq C|\lambda|^{s-\frac{3}{2}} e^{-|\bar{\rho}| \lambda^{1 / 2}}
\end{aligned}
$$

and

$$
\begin{aligned}
\left\|f_{\lambda}\right\|_{H^{s}}\left(\lambda^{-1 / 2}\right) & =|\lambda|^{s-\frac{3}{2}}\|f\|_{H^{s}}(0) \\
& \geq|\lambda|^{s-\frac{3}{2}} C \sum|\xi|^{1+\gamma+\zeta}\left|\hat{f}_{0}(\xi)\right| \\
& \geq \infty
\end{aligned}
$$

for $s>3 / 2$ and $\gamma, \zeta$ small enough. This shows that the problem is ill-posed for $s>3 / 2$ taking $\lambda$ large enough. 


\section{Stable case}

In this section we show some analytic properties and numerical simulations of the stable case. We give the main ideas of the proof of local well-posedness. We show the conservation of mass property of the equation. We give a decay estimate on the $L^{\infty}$ norm of the interface. Finally, we illustrate three numerical examples in which the analytical results are verified.

4.1. Well-posedness. In [6] we give the proof of the following result:

Theorem 4.1. Let $f_{0} \in H^{s}(\mathbb{T})$ for $s \geq 3$ and $\bar{\rho}>0$. Then there exists a time $T>0$ so that there is a unique solution $f$ of $(5)$ in $C^{1}\left([0, T] ; H^{s}(\mathbb{T})\right)$ such that $f(\alpha, 0)=f_{0}(\alpha)$.

Here we present the main ideas of the proof by using an approximation of the equation (4) given by

$$
\begin{aligned}
f_{t}(\alpha, t) & =-\bar{\rho} \frac{1}{1+\left(\partial_{x} f(\alpha, t)\right)^{2}} \Lambda f(\alpha, t), \\
f(\alpha, 0) & =f_{0}(\alpha) .
\end{aligned}
$$

The equation (9) is obtained from (4) by approximating $(f(\alpha, t)-f(\alpha-\beta, t))^{2}$ in the singular integral by $\left(\partial_{\alpha} f(\alpha, t) \beta\right)^{2}$. This equation is more local than the original system, therefore it is easier to prove existence and uniqueness. We obtain easily that

$$
\frac{d}{d t}\|f\|_{L^{2}}^{2}(t) \leq C\|f\|_{L^{2}}(t)\|\Lambda f\|_{L^{2}}(t) \leq C\|f\|_{H^{1}}^{2}(t) .
$$

Taking three derivatives in (9), and using Sobolev inequalities, we obtain for $k>2$

$$
\frac{d}{d t}\left\|\partial_{\alpha}^{3} f\right\|_{L^{2}}^{2}(t) \leq C\|f\|_{H^{3}}^{k}(t)+I_{1}+I_{2}
$$

where the most singular terms are

$$
I_{1}=\bar{\rho} \int \frac{\Lambda f(\alpha, t) \partial_{\alpha} f(\alpha, t)}{\left(1+\left(\partial_{\alpha} f(\alpha, t)\right)^{2}\right)^{2}} \partial_{\alpha}^{3} f(\alpha, t) \partial_{\alpha}^{4} f(\alpha, t) d \alpha
$$

and

$$
I_{2}=-\bar{\rho} \int \frac{1}{1+\left(\partial_{\alpha} f(\alpha, t)\right)^{2}} \partial_{\alpha}^{3} f(\alpha, t) \Lambda \partial_{\alpha}^{3} f(\alpha, t) d \alpha .
$$

We can control $I_{1}$ by integrating by parts. The $I_{2}$ term is estimated by using the following pointwise estimate (see [4])

$$
g(\alpha) \Lambda g(\alpha)-\frac{1}{2} \Lambda\left(g^{2}\right)(\alpha) \geq 0,
$$

for a general function $g(\alpha)$. It yields (suppressing the time variable)

$$
\begin{aligned}
I_{2} & =-\bar{\rho} \int \frac{\partial_{\alpha}^{3} f(\alpha) \Lambda \partial_{\alpha}^{3} f(\alpha)-\frac{1}{2} \Lambda\left(\left(\partial_{\alpha}^{3} f\right)^{2}\right)(\alpha)}{1+\left(\partial_{\alpha} f(\alpha)\right)^{2}} d \alpha-\frac{\bar{\rho}}{2} \int \Lambda\left(\frac{1}{1+\left(\partial_{\alpha} f(\cdot)\right)^{2}}\right)(\alpha)\left(\partial_{\alpha}^{3} f(\alpha)\right)^{2} d \alpha \\
& \leq C\left\|\Lambda\left(\frac{1}{1+\left(\partial_{x} f(\cdot)\right)^{2}}\right)\right\|_{L^{\infty}}\left\|\partial_{\alpha}^{3} f\right\|_{L^{2}}^{2} \\
& \leq C\|f\|_{H^{3}}^{k}
\end{aligned}
$$

by Sobolev estimates. Finally we have that

$$
\frac{d}{d t}\|f\|_{H^{3}}^{2}(t) \leq C\|f\|_{H^{3}}^{k}(t)
$$


for $C$ and $k>2$ fixed constants. Integrating in time we obtain existence.

Integrating in a similar way, we have that for two solutions of the system (9), $f_{1}$ and $f_{2}$ satisfy

$$
\frac{d}{d t}\left\|\left(f_{1}-f_{2}\right)\right\|_{L^{2}}^{2}(t) \leq C\left(f_{1}, f_{2}\right)\left\|\left(f_{1}-f_{2}\right)\right\|_{L^{2}}^{2}(t),
$$

and using Gronwall's inequality we get uniqueness.

4.2. Mass conservation. We show that the mean of the function $f(\alpha, t)$ is conserved. The equation (6) can be written as follows

$$
f_{t}(\alpha, t)=\frac{\bar{\rho}}{\pi} \partial_{\alpha} \int_{\mathbb{T}} \arctan \left(\frac{\tanh ((f(\alpha, t)-f(\alpha-\beta, t)) / 2)}{\tan (\beta / 2)}\right) d \beta
$$

and it gives

$$
\int_{\mathbb{T}} f(\alpha, t) d \alpha=\int_{\mathbb{T}} f_{0}(\alpha) d \alpha
$$

This property of mass conservation is satisfied in the numerical experiment performed in section 4.4 .

4.3. Decay for the $L^{\infty}$ norm. Here we show that the $L^{\infty}$ norm of the solution of the system is bounded in time. We also get for any initial data a global in time estimate for the evolution of the maximum and the minimum of the contour $(\alpha, f(\alpha, t))$.

For $f_{0} \in H^{s}(\mathbb{T})$ with $s \geq 3$, there exists a time $T>0$ such that the solution of (6) $f(\alpha, t)$ belongs to $C^{1}\left([0, T] ; H^{s}(\mathbb{T})\right)$ and in particular to $C^{1}([0, T] \times \mathbb{T})$. Therefore, using the Rademacher theorem, the function

$$
M(t)=\max _{\alpha} f(\alpha, t)
$$

is differentiable for almost every $t$. Let $\alpha_{t}$ satisfy $0<f\left(\alpha_{t}, t\right)=M(t)$ (a similar argument can be used for the minimum $\left.m(t)=f\left(\bar{\alpha}_{t}, t\right)>0\right)$.

If we consider a point in which $M(t)$ is differentiable, we have

$$
M^{\prime}(t)=f_{t}\left(x_{t}, t\right)
$$

(see [5] or [7] for more detail). The formula (10) gives

$$
f_{t}(\alpha, t)=\frac{\bar{\rho}}{\pi} \partial_{\alpha} \int_{\mathbb{T}} \arctan \left(\frac{\tanh ((f(\alpha, t)-f(\beta, t)) / 2)}{\tan ((\alpha-\beta) / 2)}\right) d \beta
$$

and therefore

$$
\begin{aligned}
& f_{t}(\alpha, t)=\frac{\bar{\rho}}{2 \pi} \partial_{\alpha} f(\alpha, t) P V \int_{\mathbb{T}} \frac{\tan ((\alpha-\beta) / 2)\left(1-\tanh ^{2}((f(\alpha, t)-f(\beta, t)) / 2)\right)}{\tan ^{2}((\alpha-\beta) / 2)+\tanh ^{2}((f(\alpha, t)-f(\beta, t)) / 2)} d \beta \\
&-\frac{\bar{\rho}}{2 \pi} P V \int_{\mathbb{T}} \frac{\tanh ((f(\alpha, t)-f(\beta, t)) / 2)\left(1+\tan ^{2}((\alpha-\beta) / 2)\right)}{\tan ^{2}((\alpha-\beta) / 2)+\tanh ^{2}((f(\alpha, t)-f(\beta, t)) / 2)} d \beta .
\end{aligned}
$$

Using the above equation evaluated at $\alpha=\alpha_{t}$ and the fact that $\partial_{\alpha} f\left(\alpha_{t}, t\right)=0$, we obtain

$$
M^{\prime}(t)=-\frac{\bar{\rho}}{2 \pi} P V \int_{\mathbb{T}} \frac{\tanh ((M(t)-f(\beta, t)) / 2)\left(1+\tan ^{2}((\alpha-\beta) / 2)\right)}{\tan ^{2}((\alpha-\beta) / 2)+\tanh ^{2}((f(\alpha, t)-f(\beta, t)) / 2)} d \beta,
$$

and we get $M^{\prime}(t) \leq 0$ for almost every $t$. Integrating in time we obtain $M(t) \leq M(0)$. Analogously we have for the minimum that $m(t) \geq m(0)$. This concludes the argument to achieve the following maximum principle: 
Lemma 4.2. If $f_{0} \in H^{s}(\mathbb{T})$ with $s \geq 3$, then the unique solution of the system (6) satisfies

$$
\|f\|_{L^{\infty}}(t) \leq\left\|f_{0}\right\|_{L^{\infty}} .
$$

In fact $M(t) \leq M(0)$ and $m(t) \geq m(0)$.

We notice that for the model (9), the same result is obtained using this argument and the following formula for the operator $\Lambda$ (see [4]):

$$
\Lambda g(\alpha, t)=\frac{1}{4 \pi} P V \int_{\mathbb{T}} \frac{g(\alpha)-g(\beta)}{\sin ^{2}((\alpha-\beta) / 2)} d \beta .
$$

The above lemma provides the next result.

Lemma 4.3. Let $f_{0} \in H^{s}(\mathbb{T})$ with $s \geq 3$, then the maximum $M(t)$ and the minimum $m(t)$ of the solution of the system (6) satisfy

$$
M(t)-\frac{1}{2 \pi} \int_{\mathbb{T}} f_{0}(\alpha) d \alpha \leq M(0) e^{-C t}
$$

and

$$
m(t)-\frac{1}{2 \pi} \int_{\mathbb{T}} f_{0}(\alpha) d \alpha \geq m(0) e^{-C t},
$$

for $C=\bar{\rho} / 2 \cosh ((M(0)-m(0)) / 2))$.

Using the formula (11), the estimate

$$
\tan ^{2}((\alpha-\beta) / 2)+\tanh ^{2}((f(\alpha, t)-f(\beta, t)) / 2) \leq \tan ^{2}((\alpha-\beta) / 2)+1
$$

yields

$$
M^{\prime}(t) \leq-\frac{\bar{\rho}}{2 \pi} P V \int_{\mathbb{T}} \tanh ((M(t)-f(\beta, t)) / 2) d \beta .
$$

The maximum principle gives the following inequality

$$
\cosh ((M(t)-f(\beta, t)) / 2) \leq \cosh ((M(0)-m(0)) / 2)
$$

and taking

we obtain

$$
\sinh ((M(t)-f(\beta, t)) / 2) \geq(M(t)-f(\beta, t)) / 2,
$$

$$
M^{\prime}(t) \leq-C M(t)+\frac{C}{2 \pi} \int_{\mathbb{T}} f(\beta, t) d \beta .
$$

The mass conservation property and integrating in time give the estimate (12). In a similar way (13) is obtained.

4.4. Numerical simulations. In this section, we show some simulations of the dynamics of the interface in porous media. We approximate the solutions of (6) with three different initial data and $\bar{\rho}=2 \pi$.

Now, we discuss the numerical method used in the simulations. The evolution is calculated by using ideas developed in [9] for the case of the 2D Euler equations. In particular, contours are represented by an adaptive node spacing with $N$ nodes adjusted nonlocally at each time step and cubic spline interpolation between nodes. The contour integral is numerically evaluated between each two nodes using an adaptive Lobatto quadrature. Contour integrals along each cubic contour segment are evaluated as follows: series expansions on small parameters are used to evaluate the singular integrands, i.e, when the segment contains the singular point 
Figure 1. The interface $f$ in times 0, 0.5, 1 and 2, and the evolution in time of the logarithm of $L^{\infty}$-norms of $f, f_{x}$ and $f_{x x}$ in Case1.
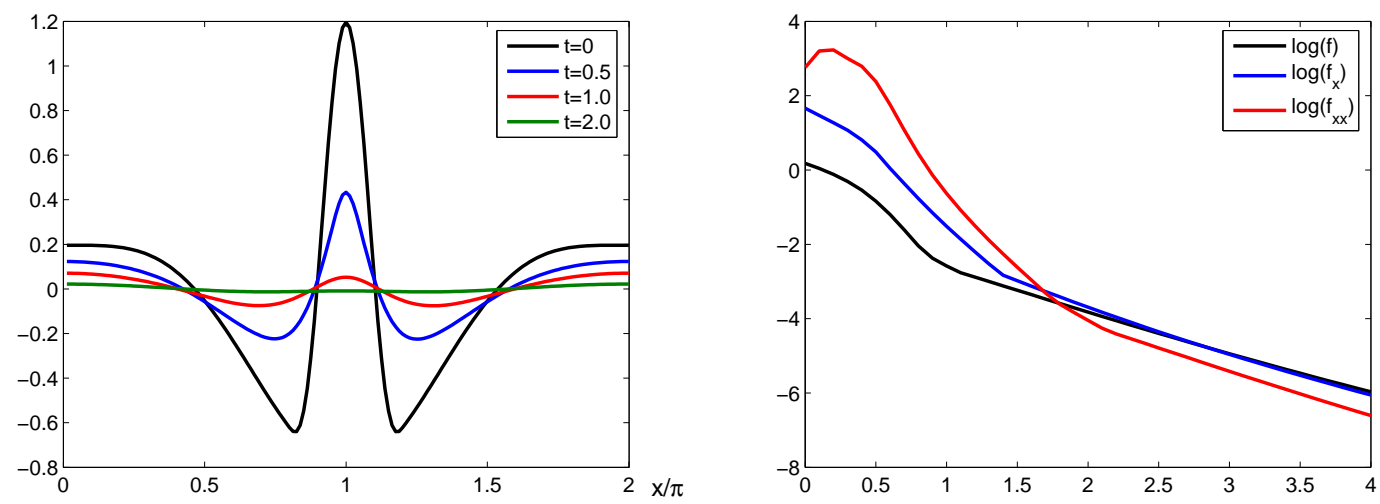

$x$; for the regular integrands the explicit functions and splines are evaluated. After the node representation of contours, the equation (6) is transformed in a set of coupled ordinary differential equations that are forward time-integrated with a fourth-order Runge Kutta method. Time step size $\tau$ is adjusted with the space step $h$. In our numerical simulations, our space step is initially fixed considering the smallest in the region where there is the highest slope.

We consider the following initial data for our simulations: two symmetric and other non symmetric.

The Case 1 is defined by the following initial datum

$$
f_{1}(x)=\left\{\begin{array}{cl}
\sigma(x), & 0<x<(0.8) \pi \\
\sigma(x)-h(x), & (0.8) \pi<x<(1.2) \pi \\
\sigma(x), & (1.2) \pi<x<2 \pi
\end{array}\right.
$$

with

$$
\sigma(x)=-\sin ^{4}(x)
$$

and

$$
h(x)=2\left(1-5\left(\frac{x}{\pi}-1\right)^{2}\right)^{3} .
$$

The initial datum of Case 2 is given by

$$
f_{2}(x)=\left\{\begin{array}{cl}
\sin ^{3}\left(\frac{x-x_{0}}{2 h_{0}}\right), & x_{0}<x<x_{1} \\
1, & x_{1}<x<x_{2} \\
\sin ^{3}\left(\frac{x_{3}-x}{2 h_{0}}\right), & x_{2}<x<x_{3} \\
0, & \text { otherwise }
\end{array}\right.
$$

where $x_{0}=\pi\left(1-h_{0}\right) / 2, x_{1}=\pi\left(1+h_{0}\right) / 2, x_{2}=\pi\left(3-h_{0}\right) / 2$ and $x_{3}=\pi\left(3+h_{0}\right) / 2$ with $h_{0}=1 / 8$.

Finally, the initial datum of Case 3 satisfies

$$
f_{3}(x)=\left\{\begin{array}{cl}
a \sin ^{3}(x), & 0<x<\pi, \\
-b \sin ^{3}(x), & \pi<x<2 \pi,
\end{array}\right.
$$


Figure 2. The interface $f$ in times 0,1 and 2, and the evolution in time of the logarithm of $L^{\infty}$-norms $f, f_{x}$ and $f_{x x}$ in Case 2.
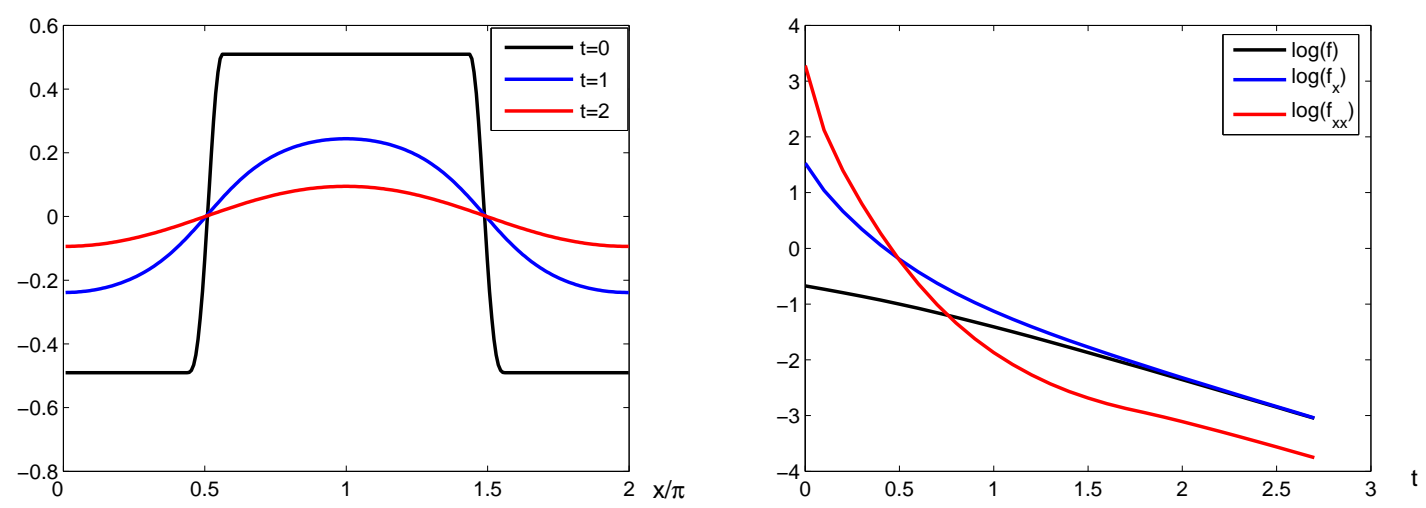
with $a=0.85$ and $b=2.25$.

The space step of the simulations is $h=2 \pi / N$ with $N=160$ in Case $1, N=256$ in Case 2 and $N=192$ in Case 3. The time step is $\tau=0.01$ in Case 1 and $\tau=0.005$ in the other cases. Other time steps and mesh sizes have been used with similar results. The approximations have order 2 in space and order 4 in time.

In section 4.2, we prove that the solution of (6) satisfies the law of mass conservation. In our numerical scheme, the mass conservation is ensured with order 4 . The masses, denoted by $\bar{f}$

$$
\bar{f}(t)=\frac{1}{2 \pi} \int_{0}^{2 \pi} f(x, t) d x,
$$

of the different cases are: $\bar{f}=-0.1961$ in Case $1 ; \bar{f}=0.5$ in Case 2 ; and $\bar{f}=-0.6578$ in Case 3. In our figures we present the evolution of $f-\bar{f}$ in different times.

Other natural theoretical property shown in the simulations is the decay for the $L^{\infty}$ norm (see section 4.3). Moreover, we find that the decay of the slope and the curvature are exponential. Indeed, in the second picture of the figures we plot the logarithm of the $L^{\infty}$ norm of the difference of the contour function and their mass. It is denoted by $\log (f)$, i.e.,

$$
\log (f)(t)=\log \left(\max _{x}|f(x, t)-\bar{f}|\right) .
$$

We notice in the figures that the decay of $\log (f)$ is linear after any time.

By $\log \left(f_{x}\right)$ and $\log \left(f_{x x}\right)$ we denote the logarithm of the $L^{\infty}$-norm of the contour slope and the contour curvature, i.e.,

$$
\log \left(f_{x}\right)=\log \left(\max _{x}\left|f_{x}(x, t)\right|\right),
$$

and

$$
\log \left(f_{x x}\right)=\log \left(\max _{x}\left|f_{x x}(x, t)\right|\right) .
$$

In section 4.3 we proved that the $L^{\infty}$-norm of $f$ decay as an exponential. In the graphics, we clearly show exponential decay in the $L^{\infty}$-norm of $f$ and, also, in the $L^{\infty}$-norm of $f_{x}$ and $f_{x x}$. In particular, this norm decays as $e^{-\delta t}$ with $\delta=1.103$ in Case $1, \delta=0.987$ in Case 2 and $\delta=1.14$ in Case 3 .

It is clear that these numerical solutions indicate a regularity effect. Indeed, the decay of the slope and the curvature is stronger than the maximum of $f-\bar{f}$. Thus, the irregular regions in our graphics are rapidly smoothed and the flat regions are smoothly bend (see Figure 2). This fact is observed easily in the Case 1 and 2. The initial maximum is at $x_{m}=\pi$ and, on the other hand, $x_{0}=0$ is a relative maximum. However, around $x_{m}$ there is a strong irregular region with respect to $x_{0}=0$. Thus, we observe that the decay of $f\left(x_{m}, t\right)$ is faster than $f\left(x_{0}, t\right)$ and rapidly $x_{0}$ becomes the maximum point.

The computational part of this work was performed on ODISEA (cluster of 16 dual nodes in Rack with 2 processors Intel Xeon EMT64 3.2 GHz) of the Group of Research in Mathematical Modeling and Numerical Simulation in Science and Technology (SIMUMAT) supported by the grant S-0505/ESP/0158 of the Council of Education of the Regional Government of Madrid (Spain). We used the MATLAB routines to obtain the calculations.

\section{Conclusions}

In this article we describe the evolution of a free boundary that evolves by an incompressible fluid in which the velocity is related to the density by singular integral operators. This 
Figure 3. The interface $f$ in times 0, 0.5, 1 and 2, and the evolution in time of the logarithm of $L^{\infty}$-norms of $f, f_{x}$ and $f_{x x}$ in non-symmetric case, Case 3.
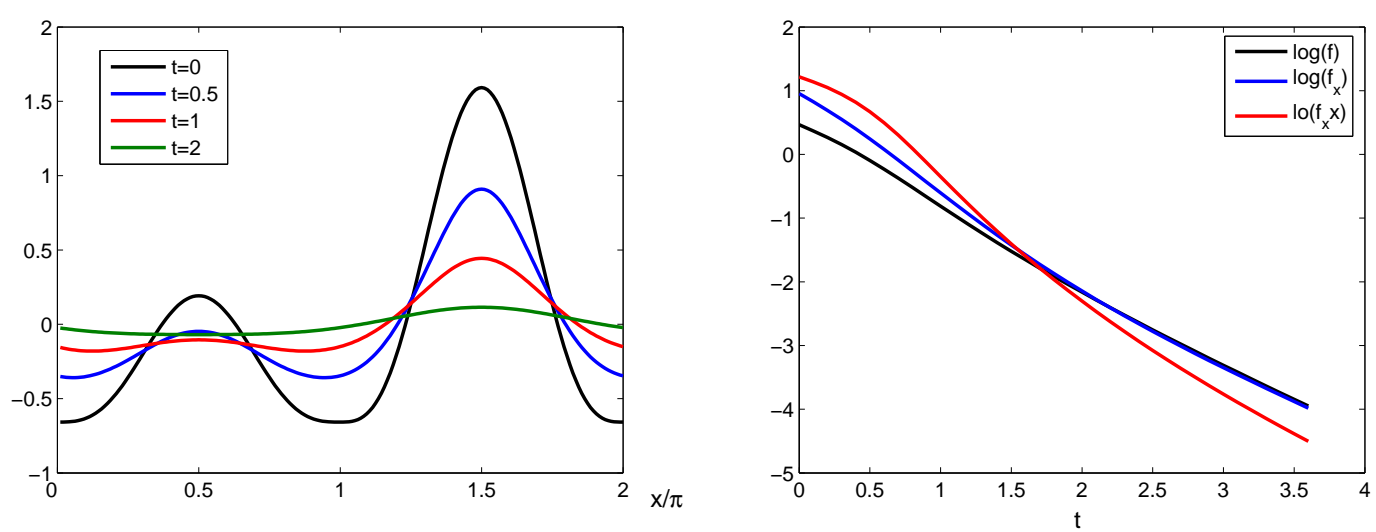
set of equations models different physical phenomena (Hele-Shaw flow, Muskat problem and convection in porous media). By using an appropriate parameterization, we avoid an important kind of singularity in the fluid when the interface collapses. A roll-up effect implies blow-up on the derivatives of the free boundary with this formulation, but in the stable case (in which the problem is well-posed) we observe a strong smoothing effect in the equation with no viscosity or surface tension involve.

\section{REFERENCES}

[1] D. Ambrose. Well-posedness of Two-phase Hele-Shaw Flow without Surface Tension. Euro. Jnl. of Applied Mathematics 15 597-607 (2004).

[2] J. Bear, Dynamics of Fluids in Porous Media, American Elsevier, New York, 1972.

[3] P. Constantin and M. Pugh. Global solutions for small data to the Hele-Shaw problem. Nonlinearity, 6, 393 - 415 (1993).

[4] A. Córdoba and D. Córdoba. A pointwise estimate for fractionary derivatives with applications to partial differential equations. Proc. Natl. Acad. Sci. USA 100, no. 26, 15316-15317 (2003).

[5] A. Córdoba and D. Córdoba. A maximum principle applied to Quasi-geostrophic equations. Comm. Math. Phys. 249 (2004), no. 3, 511-528.

[6] D. Córdoba and F. Gancedo. Contour dynamics of incompressible 3-D fluids in a porous medium with different densities. Comm. Math. Phys, 273, no. 2, 445-471 (2007).

[7] D. Córdoba and F. Gancedo. A maximum principle for the Muskat problem for fluids with different densities. Preprint, arXiv:0712.1090v1.

[8] D. Córdoba, F. Gancedo \& R. Orive. Analytical behaviour of 2D incompressible flow in porous media $J$. Math. Phys. 48, no. 6 (2007).

[9] D.G. Dritschel, Comput. Phys. Rep. 10, 77-146 (1989).

[10] T. Dombre, A. Pumir and E. Siggia. On the interface dynamics for convection in porous media. Physica $D, 57,311-329$ (1992).

[11] J. Escher and G. Simonett. Classical solutions for Hele-Shaw models with surface tension. Adv. Differential Equations, 2:619-642 (1997).

[12] Hele-Shaw. The flow of water.Nature, 58, no.1489, 34-36, 520 (1898).

[13] T.Y. Hou, J.S. Lowengrub and M.J. Shelley. Removing the Stiffness from Interfacial Flows with Surface Tension. J. Comput. Phys., 114: 312-338 (1994).

[14] M. Muskat. The flow of homogeneous fluids through porous media. New York, 1937.

[15] P.G. Saffman and Taylor. The penetration of a fluid into a porous medium or Hele-Shaw cell containing a more viscous liquid. Proc. R. Soc. London, Ser. A 245, 312-329 (1958).

[16] M. Siegel, R. Caflisch and S. Howison. Global Existence, Singular Solutions, and Ill-Posedness for the Muskat Problem. Comm. Pure and Appl. Math., 57: 1374-1411 (2004).

[17] E. Stein. Singular Integrals and Differentiability Properties of Function. Princeton University Press. Princeton, NJ, 1970.

Diego CóRdoba

Instituto de Ciencias Matemáticas

Consejo Superior de Investigaciones Científicas

Serrano 123, 28006 Madrid, Spain.

E-mail address: dcg@imaff.cfmac.csic.es

Francisco Gancedo

Department of Mathematics

UNIVERSITY OF CHICAGO

5734 University Avenue, ChicAgo, IL 60637, USA.

E-mail address: fgancedo@math.uchicago.edu 


\section{RAFAel Orive}

Departamento de Matemáticas

Facultad de Ciencias

Universidad Autónoma de Madrid

Crta. Colmenar Viejo Km. 15, 28049 Madrid, Spain.

E-mail address: rafael.orive@uam.es 